\title{
COMPACTNESS OF THE MASSIVE SEIBERG-WITTEN EQUATION*
}

\author{
NAICHUNG CONAN LEUNG ${ }^{\dagger}$ AND MING XU
}

Key words. 4-manifold, compactness theorem, massive Seiberg-Witten equation, massive Seiberg-Witten moduli space.

AMS subject classifications. 57R 57

In 1994, the Seiberg-Witten (SW) equation was introduced by Witten [W]. This system couples the anti-self-dual (ASD) equation for $U(1)$-con-nections with a harmonic spinor on four manifolds. By counting the number of its solutions, one can define the SW invariants. Conjecturally, the SW invariants are equivalent to the Donaldson invariants for four manifolds. Unlike the ASD equation for $S U(2)$-connections in the Donaldson theory, the SW equation has an amazing property which makes the SW theory much easier. Namely, the moduli space of solutions to the SW equation is compact.

In this article, we generalize the SW equation by allowing the spinor to have mass or energy level up to level $n$. We show that this massive SW equation also has compactness property. We will use the moduli space of mSWequation to define invariants for four manifolds in $[\mathrm{LX}]$.

To prove our compactness result, we again need to use the Weitzenböck formula and bootstrapping arguments as in the original SW theory. However new ingredients are needed here. These include a eigenvalue estimate by Vafa and Witten, a repeated use of the Weitzenböck formula and a control of individual eigencomponent of the spinor field.

1. Brief review of Seiberg-Witten theory. For any smooth compact closed oriented 4-manifold $M$, with given Riemannian metric and $\operatorname{spin}^{c}$ structure, the Seiberg-Witten equation is defined for a $\operatorname{spin}^{c}$ connection $A$ and a positive spinor section $\phi$,

$$
\begin{aligned}
D_{A} \phi & =0, \\
F_{A}^{+} & =\sigma(\phi),
\end{aligned}
$$

in which $D_{A}: \Gamma\left(S^{+}\right) \rightarrow \Gamma\left(S^{-}\right)$is the Dirac operator and

$$
\sigma(\phi)=\phi \otimes \phi^{*}-\frac{|\phi|^{2}}{2} \operatorname{Id} \in \Gamma\left(\operatorname{ad}\left(S^{+}\right)\right)=\Omega_{+}^{2}(M ; i \mathbb{R})
$$

can be identified with an imaginary valued self-dual 2 -form.

The Seiberg-Witten moduli space is the quotient of the solution space of the Seiberg-Witten equation by the gauge group $\operatorname{Map}\left(M, S^{1}\right)$. We have the following compactness theorem

Theorem 1. [KM][W] The Seiberg-Witten moduli space is compact.

\footnotetext{
*Received December 10, 2008; accepted for publication August 27, 2009.

${ }^{\dagger}$ The Institute of Mathematical Sciences, The Chinese University of Hong Kong, Shatin, Hong Kong (leung@ims.cuhk.edu.hk).

${ }_{\ddagger}$ Mathematics Department, Tsinghua University, Beijing, 100084, P. R. China (mgxu@math. tsinghua.edu.cn).
} 
This compactness theorem is one of the most fundamental and important theorem in the whole theory. Because of the compactness of the moduli space, the SeibergWitten invariant can be easily defined, and it turns out to be a powerful tool for the study of 4-manifolds [D].

Let's briefly review the key steps in the proof of the above compactness theorem. We fix a smooth base connection $A_{0}$. By the Coulomb gauge fixing, we can assume the pair $(A, \phi)$ satisfies

$$
d^{*}\left(A-A_{0}\right)=0 \text { and the harmonic part of } A-A_{0} \text { is bounded. }
$$

Applying the Weitzenböck formula

$$
D_{A}^{*} D_{A} \phi=\nabla_{A}^{*} \nabla_{A} \phi+\frac{s}{4} \phi+\frac{1}{2} F_{A}^{+} \cdot \phi,
$$

in which $s$ is the scalar curvature of $M$, we can get an inequality

$$
\Delta\left(|\phi|^{2}\right)+\frac{1}{4}|\phi|^{4}+\frac{s}{2}|\phi|^{2} \leq 0 .
$$

The maximum principle provides us a pointwise estimate, i.e. an $L^{\infty}$ bound for $\phi$ which only depends on the metric. Using (2) and the gauge fixing conditions (4) on $A$, we also get an $L^{\infty}$ estimate for $A$.

Next we use bootstrapping arguments to get bounds for $(A, \phi)$ in the Sobolev spaces $L^{k, p}$ for all $k$ and $p$. Because $(A, \phi)$ is bounded in $L^{\infty}$, we have $L^{p}$ bounds for it for all $p>>0$. Use the regularity theorem of elliptic operators and the Sobolev imbedding theorem, we can get an $L^{1, p / 2}$ bound for $(A, \phi)$. The appearance of $p / 2$ instead of $p$ is due to the quadratic term in the Seiberg-Witten equation. Then similar arguments give an $L^{2, p / 4}$ bound for $(A, \phi)$. Repeat this process, we can obtain $L^{k, p / 2^{k}}$ bounds for $(A, \phi)$ for all $k$ and $p$ and prove the compactness theorem. At the same time, we can find a smooth representative for any point in the Seiberg-Witten moduli space.

The details of the proof can be found in [N2]. The Sobolev imbedding theorem and the regularity theorem for elliptic operators will be used repeatedly. The statements for those theorems are included in the Appendix.

2. Massive Seiberg-Witten equation. Let $M$ be a smooth compact closed oriented 4-manifold with the Riemannian metric $g$ and the $\operatorname{spin}^{c}$-structure $\theta$. For any spin $^{c}$-connection $A$ for $\theta$, the square of the Dirac operator

$$
D_{A}{ }^{*} D_{A}: \Gamma\left(S^{+}\right) \rightarrow \Gamma\left(S^{-}\right) \rightarrow \Gamma\left(S^{+}\right)
$$

has eigenvalues $0=\lambda_{0}(A)<\lambda_{1}(A) \cdots<\lambda_{m}(A)<\cdots$, with multiplicities $n_{0}(A)$, $n_{1}(A), \ldots, n_{m}(A), \ldots$, and we write the eigenspace decomposition as

$$
\Gamma\left(S^{+}\right)=\Gamma_{0}(A) \oplus \Gamma_{1}(A) \oplus \cdots \oplus \Gamma_{m}(A) \oplus \cdots .
$$

By our convention, all $n_{i}>0$ except $n_{0}$ can be 0 .

For any nonnegative $n$, we define $m(n, A)$ to be the smallest nonnegative integer $m$ such that $n_{0}(A)+n_{1}(A)+\cdots+n_{m}(A) \geq n+\operatorname{ind} D_{A}$. Let $H_{n, A}$ be the orthogonal projection from $\Gamma\left(S^{+}\right)$to $\sum_{i=m(n, A)+1}^{\infty} \Gamma_{i}(A)$. In particular,

$$
D_{A} \phi=0 \text { if and only if } H_{0, A} \phi=0 .
$$


We now define the massive Seiberg-Witten equation for a non-negative level $n$ :

Definition 2. The massive Seiberg-Witten equation of level $n$, denoted by $m S W_{n}$ for simplicity, is the following system of equations for a $\operatorname{spin}^{c}$ con-nection $A$ and a positive spinor $\phi$ :

$$
\begin{aligned}
H_{n, A} \phi & =0, \\
F_{A}{ }^{+} & =\sigma(\phi) .
\end{aligned}
$$

REMARK 3. The massive Seiberg-Witten equation of level 0 or level $n$ with $n+\operatorname{index}\left(D_{A}\right) \leq 0$ is the original Seiberg-Witten equation/W]. Here index $\left(D_{A}\right)$ is the index of the twisted Dirac operator and it can be computed using the AtiyahSinger index theorem. Loosely speaking, the massive Seiberg-Witten equation allows the spinor field to have mass or energy level up to $n$.

It can be easily checked that $\mathrm{mSW}_{n}$ is equivariant under the action of the gauge group $\operatorname{Map}\left(M, S^{1}\right)$. So we can define the massive Seiberg-Witten moduli space accordingly.

Definition 4. The massive Seiberg-Witten moduli space of level $n$, denoted by $\mathfrak{M}_{n}(M, \theta ; g)$, is the quotient of the solution set of $m S W_{n}$ by the action of the gauge $\operatorname{group} \operatorname{Map}\left(M, S^{1}\right)$.

If we list all the eigenvalues of $D_{A}^{*} D_{A}$ with multiplicities in the increasing order, we get the eigenvalue sequence of $D_{A}^{*} D_{A}$.

REMARK 5. Let $n^{\prime}=n+\operatorname{index}\left(D_{A}\right)>0$. Then the $n^{\prime}$-th term in the eigenvalue sequence of $D_{A}^{*} D_{A}$ is $\lambda_{m(n, A)}$, and it depends continuously on $A[C]$. So $m(n, A)$ is an lower semi-continuous function in $A$, i.e. there is a neighborhood $U$ of $A$ such that $m(n, A)=\min _{A^{\prime} \in U} m\left(n, A^{\prime}\right)$. These facts imply the solution set of $m S W_{n}$ is a closed subset inside the configuration space and they will be useful in section $\%$.

The main theorem in this article is the following compactness property of the Seiberg-Witten massive moduli space.

THEOREM 6. For any level n, the massive Seiberg-Witten moduli space is compact.

The proof of the main theorem will occupy the remaining sections.

3. Preparation and eigenvalue estimates. The final steps in the proof of our main theorem will need bootstrapping arguments as in the proof of Theorem 1 $[\mathrm{KM}][\mathrm{N} 2]$ for the original SW theory. With $k_{0}>2$ and $p_{0}>2$ properly chosen, we consider the $L^{k_{0}, p_{0}}$ massive moduli space, i.e. the space of $L^{k_{0}, p_{0}}$ solutions of $\mathrm{mSW}_{n}$ divided by the $L^{k_{0}+1, p_{0}}$ gauge action. The bootstrapping arguments will prove, by choosing the gauge, representatives of the moduli space can be found inside the space of smooth solutions of the $\mathrm{mSW}_{n}$, and they are bounded in any such Sobolev spaces. As a by-product, we see the massive SW moduli space does not depend on $k_{0}$ or $p_{0}$, and we need only to study the massive SW moduli space of smooth solutions.

Unlike the proof of Theorem 1, when the eigenvectors of positive eigenvalues are involved, we can not initiate the bootstrapping arguments with a $L^{\infty}$ estimate using the Weitzenböck formula. Only an $L^{1,2}$ estimate can be directly deduced. More careful estimates will give $L^{p}$ estimates for all $p>1$. Though it is weaker then $L^{\infty}$ estimates, but enough for us to start the bootstrapping arguments. 
To get the $L^{1,2}$ bound for the Seiberg-Witten solution, we need an important estimate for the eigenvalues of $D_{A}^{*} D_{A}$ by Vafa and Witten [VW][A].

Theorem 7 (Vafa-Witten). For any integer $n>0$, the $n$-th term in the eigenvalue sequence of $D_{A}^{*} D_{A}$ is bounded by a constant independent of $A$.

Here we outline a proof. Let $E$ be a complex two dimensional bundle on $M$ with $c_{1}(E)=0$ and $c_{2}(E)=N>>0$. Let $F$ be the complex two dimensional bundle with $c_{1}(F)=0$ and $c_{2}(F)=-N$. Then $E \oplus F$ is trivial bundle over $M$. There are two Hermitian connections on $E \oplus F$, the trivial one $A_{0}$ for the trivialization of $E \oplus F$, and the reduced one $A_{1}$ for the decomposition $E \oplus F$. For any spin ${ }^{c}$ connection $A$, we denote the corresponding Dirac operator on $\Gamma\left(S^{+} \otimes(E \oplus F)\right)$ for $A+A_{0}$ and $A+A_{1}$ by $D_{0}$ and $D_{1}$ respectively. Then $D_{1}-D_{0}$ is independent of $A$. For $D_{1}$, its kernel's dimension is at least the one for $\left.D_{1}\right|_{\Gamma\left(S^{+} \otimes F\right)}$, which is bounded below by $2 \operatorname{ind} D_{A}+N$. For any section $\phi \in \operatorname{ker} D_{1}$, we can get an estimate $\left\|D_{0} \phi\right\|_{L^{2}} \leq\left\|\left(D_{1}-D_{0}\right) \phi\right\|_{L^{2}} \leq C\|\phi\|_{L^{2}}$ for some universal constant $C>0$. This implies we get the universal estimates for the upper bounds of the first $2 \operatorname{ind} D_{A}+N$ eigenvalues (counting multiplicities) of $D_{0}{ }^{*} D_{0}$. At the same time, all the eigenvalues of $D_{0}{ }^{*} D_{0}$ are linear combinations of eigenvalues of $D_{A}^{*} D_{A}$ with nonnegative integer coefficients. When $N$ is big enough, the universal upper bound of the $n^{\prime}$-th term in the eigenvalue sequence for $A$ can be found.

4. The $L^{1,2}$ estimates. We will assume $n^{\prime}=n+\operatorname{ind} D_{A}>0$. During the proof of the main theorem, all bounds and estimates we get will only depend on the metric and the $\operatorname{spin}^{c}$ structure.

Let $(\phi, A)$ be a solution for $\mathrm{mSW}_{n}$. Fix a smooth background connection $A_{0}$, and use the Coulomb gauge fixing (4), i.e. $\alpha=A-A_{0} \in \operatorname{ker}\left(d^{*}\right)$, and the harmonic part of $\alpha$ is bounded. We decompose $\phi$ as

$$
\phi=\phi_{0}+\cdots+\phi_{m}
$$

with each $\phi_{i} \in \Gamma_{i}(A)$ for $i=0,1, \ldots, m$, and $m=m(n, A)$. As Remark 5 indicates, we can use the eigenvalue estimate of Vafa-Witten (Theorem 7 ) to get a universal bound $C_{1}>0$ for $\lambda_{m}(A)$. Then we have

$$
\int<D_{A}^{*} D_{A} \phi, \phi>=\int \sum_{i=0}^{m} \lambda_{i}(A)\left|\phi_{i}\right|^{2} \leq C_{1} \sum_{i=0}^{m}\left\|\phi_{i}\right\|_{L^{2}}^{2}=C_{1}\|\phi\|_{L^{2}}^{2} .
$$

Using the Weitzenböck formula, we get the inequality

$$
C_{1}\|\phi\|_{L^{2}}^{2} \geq \int<D_{A}^{*} D_{A} \phi, \phi>=\int\left(\left|\nabla_{A} \phi\right|^{2}+\frac{s}{4}|\phi|^{2}+\frac{1}{4}|\phi|^{4}\right) .
$$

This inequality gives a $L^{4}$ bound for $\phi$ and a $L^{2}$ bound for $\nabla_{A} \phi$. Applying the $L^{4}$ bound for $\phi$ to equation (2) gives an $L^{2}$ bound for $d^{+} \alpha=F_{A}^{+}-F_{A_{0}}^{+}$. Using the gauge fixing conditions (4), the elliptic estimate for the operator

$$
\left(d^{*}, d^{+}\right): \Omega^{1}(M) \rightarrow \Omega^{0}(M) \oplus \Omega_{+}^{2}(M)
$$

gives an $L^{1,2}$ bound for $\alpha$, or equivalently for $A$. Then by the Sobolev imbedding theorem for 4-manifolds, $L^{1,2}$ can be imbedded into $L^{4}$ continuously, so the $L^{1,2}$ bound also gives an $L^{4}$ bound for $A$. As $\nabla_{A_{0}} \phi=\nabla_{A} \phi-\alpha \cdot \phi$ is bounded in $L^{2}$ and $\phi$ is bounded in $L^{4}$, we get an $L^{1,2}$ bound for $\phi$ by the regularity theorem. Hence we have established the following estimate. 
LEMMA 8. There is a constant $C$ such that given any solution $(A, \phi)$ to $m S W_{n}$, we have

$$
\|\phi\|_{L^{1,2}}+\|A\|_{L^{1,2}} \leq C
$$

5. The $L^{p}$ estimates for all $p>1$. The Weitzenböck formula can not be applied to get a pointwise estimate as in the original Seiberg-Witten theory. The trick is to use that formula twice, i.e.

$$
\begin{aligned}
& \left(D_{A}^{*} D_{A}\right)^{2} \phi \\
= & D_{A}^{*} D_{A}\left(\nabla_{A}^{*} \nabla_{A} \phi+\frac{1}{4} s \phi+\frac{1}{4}|\phi|^{2} \phi\right) \\
= & \left(\nabla_{A}^{*} \nabla_{A}\right)^{2} \phi+\frac{1}{4} s \nabla_{A}^{*} \nabla_{A} \phi+\frac{1}{2} F_{A}^{+} \cdot \nabla_{A}^{*} \nabla_{A} \phi+\frac{1}{4} \nabla_{A}^{*} \nabla_{A}(s \phi)+ \\
& \frac{1}{16} s^{2} \phi+\frac{1}{16} s|\phi|^{2} \phi+\frac{1}{4} \nabla_{A}^{*} \nabla_{A}\left(|\phi|^{2} \phi\right)+\frac{1}{16} s|\phi|^{2} \phi+\frac{1}{16}|\phi|^{4} \phi .
\end{aligned}
$$

Taking $L^{2}$-inner product with $\phi$, we have a bound for the integration

$$
\int<\left(D_{A}^{*} D_{A}\right)^{2} \phi, \phi>=\sum_{i=0}^{m} \lambda_{i}(A)^{2}\left\|\phi_{i}\right\|_{L^{2}}^{2} \leq C_{1}^{2}\|\phi\|_{L^{2}}^{2}
$$

and $\int s|\phi|^{4}$ is bounded by the $L^{4}$ estimates for $\phi$. We have

$$
\int\left(<s \nabla_{A}^{*} \nabla_{A} \phi, \phi>+<\nabla_{A}^{*} \nabla_{A}(s \phi), \phi>\right)=2 \operatorname{Re} \int s<\nabla_{A}^{*} \nabla_{A} \phi, \phi>,
$$

and

$$
\begin{aligned}
& \int\left(<\frac{1}{2} F_{A}^{+} \cdot \nabla_{A}^{*} \nabla_{A} \phi, \phi>+\frac{1}{4}<\nabla_{A}^{*} \nabla_{A}\left(|\phi|^{2} \phi\right), \phi>\right) \\
= & \frac{1}{4} \int\left(<\nabla_{A}^{*} \nabla_{A} \phi,|\phi|^{2} \phi>+<|\phi|^{2} \phi, \nabla_{A}^{*} \nabla_{A} \phi>\right) \\
= & \frac{1}{2} \operatorname{Re} \int|\phi|^{2}<\nabla_{A}^{*} \nabla_{A} \phi, \phi>.
\end{aligned}
$$

So from (11) we get an inequality

$$
C_{2} \operatorname{Re} \int s<\nabla_{A}^{*} \nabla_{A} \phi, \phi>+C_{3} \operatorname{Re} \int|\phi|^{2}<\nabla_{A}^{*} \nabla_{A} \phi, \phi>\leq C_{4},
$$

in which $C_{2}, C_{3}, C_{4}$ are positive constants.

In the left hand side of (12),

$$
\begin{aligned}
\operatorname{Re} \int s<\nabla_{A}^{*} \nabla_{A} \phi, \phi> & =\int s\left|\nabla_{A} \phi\right|^{2}+\operatorname{Re} \int<\nabla_{A} \phi, \nabla s \otimes \phi> \\
& \geq-C_{5}\left\|\nabla_{A} \phi\right\|_{L^{2}}-C_{6}\left\|\nabla_{A} \phi\right\|_{L^{2}}\|\phi\|_{L^{2}} \\
& \geq-C_{7}
\end{aligned}
$$

in which $C_{5}, C_{6}$ and $C_{7}$ are positive constants. Applying the Kato inequality

$$
\Delta\left(|\phi|^{2}\right) \leq 2 \operatorname{Re}<\nabla_{A}^{*} \nabla_{A} \phi, \phi>
$$


we get

$$
\operatorname{Re} \int|\phi|^{2}<\nabla_{A}^{*} \nabla_{A} \phi, \phi>\geq \int|\phi|^{2} \Delta\left(|\phi|^{2}\right)=\int\left|\nabla\left(|\phi|^{2}\right)\right|^{2}
$$

Applying these estimates to (12), we get an $L^{2}$ bound for $\nabla\left(|\phi|^{2}\right)$. Combining with the $L^{4}$ bound for $\phi$, we get an $L^{1,2}$ bound for $|\phi|^{2}$. The Sobolev imbedding theorem gives a $L^{4}$ bound for $|\phi|^{2}$. Using (2) and the gauge fixing conditions (4), we have an $L^{1,4}$ bound for $A$. By the Sobolev imbedding theorem for 4 -manifolds again, $L^{1,4}$ can be imbedded into $L^{p}$ bounds for all $p>1$, so the $L^{1,4}$ bound gives bounds in all $L^{p}$ 's for $A$.

Next we will get $L^{p}$ bounds for $\phi$ for any $p>1$. Comparing $D_{A}^{*} D_{A}$ and $D_{A_{0}}^{*} D_{A_{0}}$, we have

$$
D_{A_{0}}^{*} D_{A_{0}} \phi=D_{A}^{*} D_{A} \phi-|\alpha|^{2} \phi+2 \nabla_{v(\alpha)} \phi-\left(d^{+} \alpha\right) \cdot \phi,
$$

in which $v(\alpha)$ is the imaginary valued tangent field dual to $\alpha$ by the metric. Among the terms of the right side of (14), $\nabla_{v(\alpha)} \phi$ has an $L^{2-\epsilon}$ bound for any positive $\epsilon$, and the others all have $L^{2}$ bounds. So the regularity theorem for $D_{A_{0}}^{*} D_{A_{0}}$ gives an $L^{2,2-\epsilon}$ bound for $\phi$. By the Sobolev imbedding theorem, an $L^{p}$ bound for $\phi$ for each $p>1$ follows.

Based on the $L^{p}$ estimate for $\phi$ for any $p>1$, equation (2) and the gauge fixing conditions give an $L^{1, q}$ bound for $A$ with $q=p / 2$. Let $p>8$, then we have an $L^{\infty}$ bound for $A$ by the Sobolev imbedding theorem. Hence we have established the following estimate.

LEMMA 9. For any positive $p$, there is a constant $C_{p}$ such that given any solution $(A, \phi)$ to $m S W_{n}$, we have

$$
\|\phi\|_{L^{p}}+\|A\|_{L^{p}} \leq C_{p}
$$

6. Estimates for $\phi_{i}$ and bootstrapping arguments. Recall the eigenvector decomposition $\phi=\phi_{0}+\cdots+\phi_{m}$ with $\phi_{i} \in \Gamma_{i}(A)$, for every $i \in\{0,1, \ldots, m\}$ is $L^{2}$-orthogonal, so only the $L^{2}$ bounds for all $\phi_{i}$ 's and for $D_{A}^{*} D_{A} \phi$ are automatically implied by the $L^{2}$ bound for $\phi$. If we want to use (14) and bootstrapping arguments to get $L^{k, p}$ bounds for $\phi$ and $A$ for larger $k$, we need to obtain corresponding estimates for each $\phi_{i}$ first.

Applying (14) to each $\phi_{i}$, we have

$$
D_{A_{0}}^{*} D_{A_{0}} \phi_{i}-2 \nabla_{v(\alpha)} \phi_{i}=D_{A}^{*} D_{A} \phi_{i}-|\alpha|^{2} \phi_{i}-\left(d^{+} \alpha\right) \cdot \phi_{i} .
$$

Because we have already gotten $L^{p}$ bounds for $d^{+} \alpha$ for all $p>1$, we have an $L^{2-\epsilon}$ bound for $d^{+} \alpha \cdot \phi_{i}$ for each any positive $\epsilon$. The term $D_{A}^{*} D_{A} \phi_{i}$ has an $L^{2}$ bound because of the eigenvalue estimate. We can also get an $L^{2}$ bound of $|\alpha|^{2} \phi_{i}$ easily. So we can obtain an $L^{2-\epsilon}$ estimate of the right side of (15) for every positive $\epsilon$. The regularity theorem for the elliptic operator $D_{A_{0}}^{*} D_{A_{0}}-2 \nabla_{v(\alpha)}$ gives us an estimate

$$
\left\|\phi_{i}\right\|_{L^{2,2-\epsilon}} \leq C\left(\left\|\phi_{i}\right\|_{L^{2-\epsilon}}+\left\|D_{A}^{*} D_{A} \phi-|\alpha|^{2} \phi_{i}-\left(d^{+} \alpha\right) \cdot \phi_{i}\right\|_{L^{2-\epsilon}}\right),
$$

in which $C>0$ is a constant which depends continuously on the $C^{2}$ norm of the metric and the $L^{\infty}$ norm of $\alpha[\mathrm{T}]$, so $C$ is bounded and we have an $L^{2,2-\epsilon}$ bound for 
$\phi_{i}$ for every positive $\epsilon<1$. The Sobolev imbedding theorem gives $L^{p}$ bounds for $\phi_{i}$ for all $p>1$.

The above arguments can be applied in a similar manner to prove the following lemma.

Lemma 10. Assume the integer $k \geq 1$. If $A$ has an $L^{k, p}$ bound for every $p>1$, then each $\phi_{i}$ has an $L^{k-1, p}$ bound for every $p>1$.

And we can apply similar arguments to get $L^{k, p}$ estimates for $\phi$ and $A$ with larger as in section 5 .

LEMma 11. Assume the integer $k \geq 1$. If all $\phi_{i}$ 's have $L^{k-1, p}$ bounds and $A$ has $L^{k, p}$ bounds for all $p>1$, then $\phi$ and $A$ have $L^{k+1, p}$ bounds for all $p>1$.

Proof. Because all $\phi_{i}$ 's have $L^{k-1, p}$ bounds, $\phi$ and $D_{A}^{*} D_{A} \phi=\sum_{i=1}^{m} \lambda_{i}(A) \phi_{i}$ has an $L^{k-1, p}$ bound. So the right side of the equation

$$
D_{A_{0}}^{*} D_{A_{0}} \phi=D_{A}^{*} D_{A} \phi-|\alpha|^{2} \phi+2 \nabla_{v(\alpha)} \phi-\left(d^{+} \alpha\right) \cdot \phi
$$

has an $L^{k-1, p / 2}$ bound. Applying the regularity theorem, we get an $L^{k+1, p / 2}$ bound for $\phi$. Without loss of generality, we can assume $p>>0$, the Sobolev imbedding theorem gives an $L^{k, p / 2}$ bound of $\sigma(\phi)$. Then applying regularity theorem to (2) and the gauge fixing conditions, we can get an $L^{k+1, p / 2}$ bound of $A$.

These lemmas exactly tell us how to get $L^{k, p}$ bounds for $(A, \phi)$ and all $\phi_{i}$ 's for all integer $k>0$ and all $p>1$ by mathematical induction.

We summarize all the estimates we have obtained as a corollary

COROLlaRY 12. There is constant $C_{k, p}>0$ such that for any point in the moduli space $\mathfrak{M}_{n}(M, \theta ; g)$, there is a smooth representative $(A, \phi)$ in the solution space with $\|A\|_{L^{k, p}}+\|\phi\|_{L^{k, p}}+\sum_{i=0}^{m(n, A)}\left\|\phi_{i}\right\|_{L^{k, p}}<C_{k, p}$.

7. Convergence in the massive moduli space. To finish the proof of the compactness theorem, we only need to prove $\mathfrak{M}_{n}(M, \theta ; g)$ is a closed subset in the quotient of the configuration space. In fact, by the Coulomb gauge fixing, we can apply Corollary 12 to get universal bounds of the representatives of the massive moduli space inside any Sobolev spaces, so their closure inside any Sobolev space is compact. This means, if we want to prove the massive moduli space is closed and consider a Cauchy sequence $\left[\left(A^{j}, \phi^{j}\right)\right] \in \mathfrak{M}_{n}(M, \theta ; g)$, then we can assume $\left\{\left(A^{j}, \phi^{j}\right)\right\}$ converges to $(A, \phi)$ in any Sobolev spaces and we only need to prove $(A, \phi)$ is a solution.

Equation (2) is naturally satisfied by $(A, \phi)$. Now we prove (1) is satisfied by $(A, \phi)$ also. There is a common $m=m\left(n, A^{j}\right)$ for infinitely many $j$, and the corresponding eigenvector decomposition is $\phi^{j}=\sum_{i=0}^{m} \phi_{i}^{j}$. Because we have the universal bounds for all $\phi_{i}^{j}$ 's inside all Sobolev spaces, by replacing $\left\{\left(A^{j}, \phi^{j}\right)\right\}$ by a subsequence if necessary, we can assume each $m\left(n, A^{j}\right)=m$, each sequence $\left\{\phi_{i}^{j}\right\}$ converges to $\phi_{i}$, each $\left\{\lambda_{i}\left(A^{j}\right)\right\}$ converges to $\lambda_{i}$, for any $i \in\{0,1, \ldots, m\}$. Then $\phi=\phi_{0}+\cdots+\phi_{m}$ and each $\phi_{i}$ is an eigenvector for the eigenvalue $\lambda_{i}$. If $\lambda_{m}>\lambda_{m}(n, A)(A)$, let $N=$ $n_{0}(A)+\cdots+n_{m}(n, A) \geq n$, then the position of $\lambda_{m}$ in the eigenvalue sequence is strictly after the $N$-th term. Remark 5 implies the same for $\lambda_{m}\left(A^{j}\right)$ for $j>>0$. This is a contradiction with the fact $m=m\left(n, A^{j}\right)$. So $(A, \phi)$ is of the $\mathrm{mSW}_{n}$ and we have finished the proof of theorem 6 . 
8. Appendix: The Sobolev imbedding theorem and the regularity theorem. In this appendix, we state analytic results that we used in this paper. Let $(M, g)$ be a smooth Riemannian manifold, $E$ be a smooth vector bundle on $M$ with a metric structure $h$ and a compatible connection $\nabla$. Then the metric $h$ on $E$ and the metric structure $g$ on the tangent and cotangent bundles of $M$ induce metric structures on all the bundles $T^{*} M^{\otimes m} \otimes E$. Compatible connections $\nabla^{T^{*} M^{\otimes m} \otimes E}$ on $T^{*} M^{\otimes m} \otimes E$ can be induced naturally by $\nabla$ on $E$ and the Levi-Civita connection for $g$. Let $\nabla^{m}$ be the differential operator defined by the composition of connections

$$
\nabla^{m}: \Gamma(E) \rightarrow \Gamma\left(T^{*} M \otimes E\right) \rightarrow \Gamma\left(T^{*} M^{\otimes 2} \otimes E\right) \rightarrow \cdots \rightarrow \Gamma\left(T^{*} M^{\otimes m} \otimes E\right) .
$$

Definition 13. Let $k$ be a nonnegative integer and $p>1$. Define the Sobolev space $L^{k, p}(E)$ to be the space of locally integrable sections $u$ of $E$, such that for each $i=0,1, \ldots, k$, there is a locally integrable section $v_{i}$ of $T^{*} M^{\otimes i} \otimes E, v_{i}=\nabla^{i} u$ in the weak sense, i.e. for all compactly supported smooth section $w$ of $T^{*} M^{\otimes i} \otimes E$,

$$
\int<v_{i}, w>=\int<u,\left(\nabla^{i}\right)^{*} w>
$$

when $i=0 v_{0}=u$, and all $v_{i}$ 's are $L^{p}$ integrable sections. Define the Sobolev norm

$$
\|u\|_{L^{k, p}}=\sum_{i=0}^{m}\left(\int\left|\nabla^{i} u\right|^{p}\right)^{1 / p}
$$

then the Sobolev norm $\|\cdot\|_{L^{k, p}}$ makes $L^{k, p}(E)$ a Banach space.

Though the Sobolev norm depends on the metrics and connections on $M$ and $E$, the space $L^{k, p}(E)$ and its topology does not depend on those structures.

TheOREM 14 (Sobolev imbedding theorem). Let $M$ be a smooth compact $d$ dimensional manifold and $E$ be a smooth vector bundle on $M$. For $(k, p)$ and $\left(k^{\prime}, p^{\prime}\right) \in$ $\mathbf{Z}_{+} \times(1, \infty)$, then $L^{k, p}(E)$ can be continuously imbedded into $L^{k^{\prime}, p^{\prime}}(E)$ if $k \geq k^{\prime}$ and $k-d / p \geq k^{\prime}-d / p^{\prime}$. The imbedding is compact if $k>k^{\prime}$ and $k-d / p>k^{\prime}-d / p^{\prime}$. The Sobolev space $L^{k, p}(E)$ can be compactly and continuously imbedded into $C^{k^{\prime}}(E)$ if $k-d / p>k^{\prime}$.

The Sobolev imbedding theorem also contains statements for imbedding Sobolev spaces into Hölder spaces. They are not used in this article, so the statement is not included here. For complete statements of the theorem, see e.g. [N1],[N2] or[T].

Theorem 15 (Regularity for elliptic operators). Suppose $M$ is a compact Riemannian manifold, $E$ and $F$ are smooth Riemannian vector bundles on $M$, and $L$ is a m-th order elliptic operator with smooth coefficients. The section $u \in L^{p}(E)$ is a weak solution of $L u=v \in L^{k, p}(E)$, then $u \in L^{k+m, p}(E)$ and there is a constant $C$ depending on $L$, on the bundles $E$ and $F$, and on the structures on the manifold and bundles, such that

$$
\|u\|_{L^{k+m, p}(E)} \leq C\left(\|u\|_{L^{p}(E)}+\|v\|_{L^{m, p}(F)}\right) .
$$

The complete version of regularity theorem also contains statement for Hölder estimates [N1][N2]. In the case the coefficients of the elliptic operator are not smooth, this regularity theorem is still valid. See $[\mathrm{T}]$ for precise statements. 
Acknowledgement. The research of the first author is partially supported by a RGC grant from the Hong Kong Government. The second author thanks The Institute of Mathematical Sciences in The Chinese University of Hong Kong where this work was being carried out during his visit.

\section{REFERENCES}

[A] M. AтіYAн, Eigenvalues of the Dirac operator, Springer, LNM 1111 (1985), pp. 251-260.

[C] J.B. Conway, A Course in Functional Analysis (2nd Edition), Springer-Verlag, GTM 96 (1994).

[D] S.K. Donaldson, The Seiberg-Witten Equations and 4-Manifold Topology, Bulletin of AMS, 32:1 (1996).

[KM] P. KRONhEImER And T. MrowkA, The genus of embedded surfaces in the projective plane, Math. Res. letters., (1994) pp. 797-808.

[LX] N.C. Leung AND M. XU, The massive Seiberg-Witten invariant, in preparation.

[N1] L.I. Nicolaescu, Lectures on the Geometry of Manifolds, World Sci. Pub. Co. (1996).

[N2] L.I. Nicolaescu, Notes on Seiberg-Witten Theory, AMS GSM 28.

[T] D. Gilbarg and N.S. Trudinger, Elliptic Partial Differential Equations of Second Order, Springer, CIM (2001).

[VW] C. VAFA AND E. Witten, Eigenvalue inequalities for fermions in gauge theories, CMP 95, 3 (1984), pp. 257-276.

[W] E. Witten, Monopoles and four manifolds, Math. Res. Lett., 1 (1994), pp. 809-822. 
